\title{
Maintenance of High Blood Pressure and Early Establishment of Pulsatile Blood Flow to the Spinal Cord during Thoracoabdominal Aortic Repair
}

\author{
Koji Furukawa ${ }^{1}$, Eisaku Nakamura1, Masanori Nishimura ${ }^{2}$, Hirohito Ishii ${ }^{1}$, \\ Kunihide Nakamura ${ }^{1}$ \\ ${ }^{1}$ Department of Cardiovascular Surgery, Faculty of Medicine, University of Miyazaki, Miyazaki, Japan \\ ${ }^{2}$ Department of Cardiovascular Surgery, Miyazaki Medical Association Hospital, Miyazaki, Japan \\ Email: ^koji_furukawa@med.miyazaki-u.ac.jp
}

How to cite this paper: Furukawa, K., Nakamura, E., Nishimura, M., Ishii, H. and Nakamura, K. (2018) Maintenance of High Blood Pressure and Early Establishment of Pulsatile Blood Flow to the Spinal Cord during Thoracoabdominal Aortic Repair. World Journal of Cardiovascular Surgery, 8, 175-188.

https://doi.org/10.4236/wjcs.2018.810017

Received: September 14, 2018

Accepted: October 23, 2018

Published: October 26, 2018

Copyright $\odot 2018$ by authors and Scientific Research Publishing Inc. This work is licensed under the Creative Commons Attribution International License (CC BY 4.0).

http://creativecommons.org/licenses/by/4.0/

\section{(c) (i) Open Access}

\begin{abstract}
Objectives: Despite continuous advancements in the surgical treatments for thoracoabdominal aortic aneurysms (TAAA), paraplegia remains a devastating treatment-related complication. We aimed to summarize our experience with a novel surgical strategy involving maintenance of high blood pressure and early establishment of pulsatile blood flow to the spinal cord. Materials and Methods: Between August 2011 and October 2017, 29 patients (age, $67 \pm$ 12 years) underwent open surgery for TAAA. According to the Crawford classification, two aneurysms were type I, eight were type II, 12 were type III, and seven were type IV. We used partial cardiopulmonary bypass under mild hypothermia in all patients except one. By maintaining distal aortic perfusion pressure at $60-80 \mathrm{mmHg}$ and creating the distal aortic anastomosis before visceral branch reconstruction, we established early perfusion of the hypogastric arteries with native pulsatile flow. Intraoperative spinal monitoring and cerebrospinal fluid drainage were performed in $26(90 \%)$ and $23(79 \%)$ patients, respectively. Nineteen patients $(66 \%)$ underwent reconstruction of the intercostal arteries. During perioperative management, the mean arterial pressure was kept $>80 \mathrm{mmHg}$. Results: No in-hospital deaths or acute neurological complications occurred. One patient (3.4\%) experienced delayed temporal paraplegia. During follow-up, aorta-related death occurred in only one patient, who developed prosthetic vascular graft infection but did not undergo repeat graft replacement. The 3-year freedom from aortic-related death was $95 \%$. Conclusion: Our surgical strategy involving maintenance of high blood pressure and early establishment of pulsatile flow to the spinal
\end{abstract}


cord was effective in preventing spinal cord injury following open surgery for

TAAA.

\section{Keywords}

Thoracoabdominal Aortic Aneurysm, Open Surgery, High-Blood-Pressure Maintenance, Pulsatile Flow

\section{Introduction}

Despite the rapid progress in the field of endovascular surgery, open surgery remains the standard treatment for thoracoabdominal aortic aneurysms (TAAAs) [1]. However, surgical TAAA repair continues to pose a substantial risk of severe treatment-related complications such as spinal cord injury (SCI), renal failure, gastrointestinal problems, and stroke [2]. Furthermore, the rate of improvement in surgical equipment for TAAA repair may be delayed because the incidence of TAAAs is much lower than that of other aneurysms [2]. Therefore, it is desirable to develop a dedicated TAAA repair technique that takes advantage of current technologies and yet provides acceptable outcomes.

SCI is one of the most serious complications of TAAA surgery, severely affecting prognosis and quality of life. Although many preventive and therapeutic approaches have been proposed [3]-[15], the risk of SCI-related paraplegia cannot be fully eliminated because its underlying mechanism is multifactorial. Some clinical and experimental reports have suggested that augmentation of systemic blood pressure during spinal cord ischemia increases spinal cord blood flow and decreases the risk of postoperative neurologic complications [16] [17] [18]. Distal aortic perfusion is widely expected to be helpful for increasing blood flow because it can exploit the rich collateral network present in most patients. However, distal aortic perfusion is usually non-pulsatile and of low pressure, which diminishes its usefulness for improving spinal cord perfusion [19]. Moreover, SCI may develop when aortic cross-clamping is prolonged [15]. Therefore, to reduce the risk of postoperative paraplegia, it is desirable to minimize spinal cord ischemia during TAAA repair.

In 2011, we adopted a new surgical strategy for TAAA repair, which included maintenance of high blood pressure and early establishment of pulsatile blood flow to the spinal cord. In this study, we aimed to summarize our experience with this new surgical strategy for TAAA repair, with specific focus on safety outcomes.

\section{Materials and Methods}

\subsection{Patients}

We retrospectively analyzed the records of all patients $(n=29)$ who underwent open surgery for TAAA at Miyazaki University Hospital between August 2011 
and October 2017. The Institutional Review Board of Miyazaki University Hospital approved this study and waived the requirement for informed consent for participation on account of the retrospective nature of the study. General informed consent for undergoing the recommended interventions and investigations was obtained from each patient before surgery. In our hospital, TAAA patients were indicated for open surgery if they had non-dissecting aneurysm with a maximum diameter of $>60 \mathrm{~mm}$, dissecting aneurysm with a maximum diameter of $>55 \mathrm{~mm}$, infected aneurysm, or rapidly enlarging aneurysm.

\subsection{Surgical Procedures}

Using a retroperitoneal approach, the entire thoracoabdominal aorta was exposed through the left fifth or sixth intercostal space. Cardiopulmonary bypass (CPB) was established through a femoral arterial cannula and a femoral venous cannula. Twenty-eight patients received partial CPB under mild hypothermia (rectal temperature, $32^{\circ} \mathrm{C}-34^{\circ} \mathrm{C}$ ), whereas the remaining patient received total $\mathrm{CPB}$ under deep hypothermia (nasopharyngeal temperature, $20^{\circ} \mathrm{C}$ ) because the distal aortic arch could not be adequately clamped. Following heparinization, the left lung was deflated as much as possible to prevent pulmonary hemorrhage due to manipulation of the lungs.

Figure 1 illustrates the novel surgical technique used in our hospital. Under

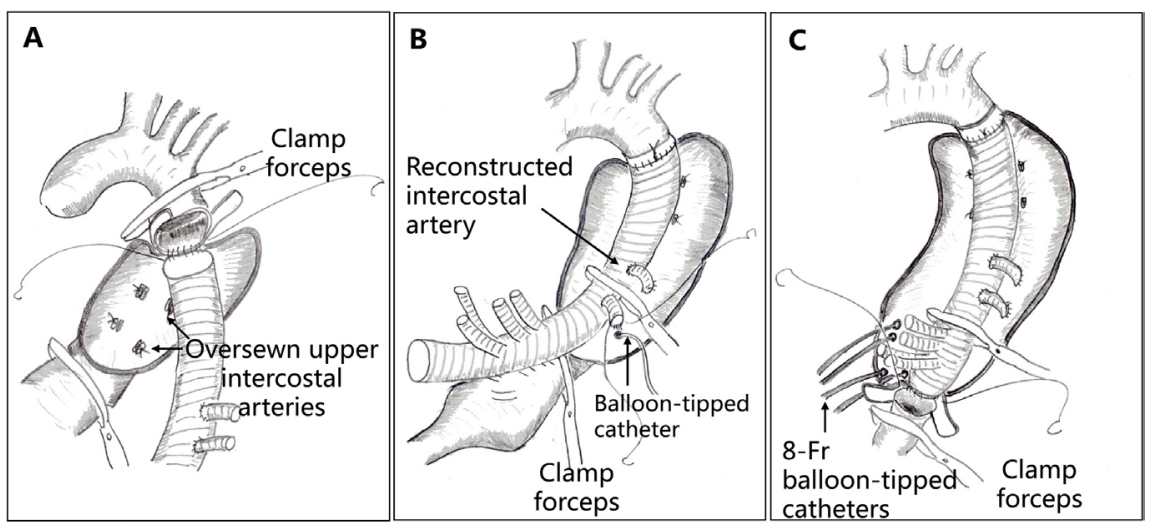

Figure 1. Novel surgical technique aiming to maximize spine perfusion during open surgery for thoracoabdominal aortic aneurysm. (A) The aorta was initially clamped just distal to the subclavian artery and at the middle of the thoracic segment (descending aorta). The orifices of the upper intercostal arteries were oversewn. The proximal aorta was completely transected, and proximal anastomosis to a Dacron graft was created. (B) The aorta just proximal to the celiac artery was clamped. Balloon-tip catheters were passed through the orifices of the intercostal and lumbar arteries at T8 to L1, and any patent intercostal and lumbar artery orifices that were relatively large and had poor backflow were selectively reconstructed according to the relative reduction in evoked potential amplitude on spinal monitoring. (C) The infra-renal portion of the abdominal aorta was clamped to maintain spinal cord perfusion as much as possible through distal perfusion. The celiac, superior mesenteric, and renal arteries were perfused selectively. Then, distal anastomosis was performed, and the lower body was perfused with native pulsatile flow. Fr French. 
partial CPB, sequential aortic clamping was performed in the cranio-caudal direction to allow the viscera and intercostal arteries to remain perfused, maintaining the distal aortic perfusion pressure at $60-80 \mathrm{mmHg}$. The aorta was initially clamped just distal to the subclavian artery and at the middle of the thoracic segment (descending aorta). Then, the aorta was incised between the clamps, and patent upper intercostal arteries were oversewn. The proximal aorta was completely transected, and proximal anastomosis to a Dacron graft was created using 4-0 polypropylene sutures with Teflon strip reinforcement (Figure $1(\mathrm{~A}))$.

Next, the aorta was clamped just proximal to the celiac artery, and the lower thoracic aorta was incised under intraoperative monitoring of spinal cord ischemia (somatosensory-evoked potential monitoring in patients treated before 2013; motor-evoked potential monitoring in patients treated from 2013 onwards). Balloon-tipped catheters were passed through the orifices of the intercostal and lumbar arteries (levels T8 through L1) in order to prevent SCI due to the steal phenomenon. Then, patent intercostal and lumbar artery orifices that were relatively large and had poor backflow [3], which was true especially in patients with evoked potential amplitude reduction to $\leq 50 \%$ of baseline on spinal monitoring [4] [5], were selectively reconstructed using an 8-mm side graft that had been previously anastomosed to the main graft at the back table. After completion of the anastomosis, the graft enabled pulsatile blood flow perfusion to the spinal cord (Figure 1(B) and Figure 1(C)).

The infra-renal portion of the abdominal aorta was clamped next. Pelvic and lower limb perfusion was retained as much as possible to maintain spinal cord perfusion. After the infra-renal portion of the abdominal aorta was opened, selective perfusion of the celiac, superior mesenteric, and renal arteries was achieved using 8-French balloon-tipped catheters via a side branch from the main CPB circuit. Upon creation of a distal anastomosis using 4-0 polypropylene sutures with Teflon stripreinforcement, perfusion of the lower body with native pulsatile flow was achieved, which increased spinal cord perfusion through the hypogastric arteries (Figure $1(\mathrm{C})$ ). Each visceral and renal artery was trimmed and reconstructed in an end-to-end manner onto the side branches of the main graft.

Thereafter, patients with evoked potential amplitude reduction to $\leq 50 \%$ of baseline despite increasing blood pressure and/or initiation of cerebrospinal fluid drainage (CSFD) underwent additional reconstruction of one or two intercostal or lumbar arteries. Intraoperative spinal monitoring and CSFD were performed in 26 (90\%) and 23 (79\%) patients, respectively.

\subsection{Perioperative Management}

During perioperative management, the mean arterial pressure was maintained $>80$ $\mathrm{mmHg}$ by regulating vasopressor levels and the intravascular volume. A CSFD catheter was placed in the lumbar region the day before the surgery, and the cerebrospinal fluid was allowed to drain freely whenever its pressure exceeded 10 
mmHg. CSFD was terminated at $48-72 \mathrm{~h}$ after surgery if the patient did not exhibit signs of SCI. Two patients with infected aneurysm underwent omentopexy following aggressive debridement and in situ graft replacement. We did not identify the Adamkiewicz artery (AKA) preoperatively.

\subsection{Follow-Up}

All patients were followed up until November 2017. The mean follow-up duration was $33 \pm 23$ months (range, 1 - 69 months).

\subsection{Statistical Analysis}

Patient characteristics, operative details, and postoperative outcome data were obtained from the clinical records. Among postoperative outcomes, we analyzed in-hospital mortality, early complications including neurological complications, the patency rate of reconstructed intercostal and lumbar arteries, aorta-related events, and late outcomes. Continuous variables are expressed as mean \pm standard deviation. The overall survival rate and the rate of freedom from aortic-related death rate were calculated using a Kaplan-Meier method that considered the period between the date of surgery and the most recent follow-up.

\section{Results}

\subsection{Patient Characteristics}

Table 1 provides a summary of the preoperative characteristics of the 29 patients included in this study (age, $67 \pm 12$ years; age range, 35 - 84 years). Three patients (10\%) had Marfan syndrome. According to the Crawford classification, two $(7 \%)$ aneurysms were type I, eight (28\%) were type II, 12 (41\%) were type III, and seven (24\%) were type IV. In terms of disease etiology, 15 (52\%) patients had atherosclerotic aneurysm, 12 (41\%) had dissecting aneurysm, and two (7\%) had infected aneurysm. Seven (24\%) patients had undergone prior abdominal aortic aneurysm (AAA) repair, including one who had also received endovascular replacement. Both hypogastric arteries were patent in 28 (97\%) patients, whereas only one hypogastric artery was patent in the remaining patient (3\%). No patient underwent emergency surgery for aortic rupture or acute aortic dissection.

\subsection{Operative Data}

Table 2 provides a summary of the operative data. The mean times spent for $\mathrm{CPB}$, aortic cross-clamping, and surgery were $178 \pm 67,112 \pm 48$, and $644 \pm 157$ min, respectively. Nineteen patients (66\%) underwent intercostal and lumbar artery reconstruction, with a total of 35 reconstructed intercostal and lumbar arteries (1.8 \pm 0.5 arteries per patient).

Among the 26 patients who underwent intraoperative spinal monitoring, reduction of the evoked potential amplitude to $\leq 50 \%$ of the baselinevalue was detected in 13 patients (50\%) (all with motor-evoked potential monitoring). In five 
Table 1. Preoperative characteristics of 29 patients who underwent open surgery for thoracoabdominal aortic aneurysm.

\begin{tabular}{cc}
\hline Variable & \\
\hline Total number & 29 \\
Age, years & $67 \pm 12$ \\
Male sex & $21(70 \%)$ \\
Marfan syndrome & $3(10 \%)$ \\
Diabetes mellitus & $3(11 \%)$ \\
CRF on HD & $2(7 \%)$ \\
Crawford classification & \\
Type I & $2(7 \%)$ \\
Type II & $8(28 \%)$ \\
Type III & $12(41 \%)$ \\
Type IV & $7(24 \%)$ \\
Etiology & \\
Atherosclerosis & $15(52 \%)$ \\
Dissection & $12(41 \%)$ \\
Infection & $2(7 \%)$ \\
Prior AAA repair & $7(24 \%)$
\end{tabular}

$C R F$ chronic renal failure, $H D$ hemodialysis, $A A A$ abdominal aortic aneurysm. Data are given as mean \pm standard deviation or frequency (percentage), as appropriate.

Table 2. Operative data of 29 patients who underwent open surgery for thoracoabdominal aortic aneurysm.

\begin{tabular}{cc}
\hline Variable & \\
\hline CPB time, min & $178 \pm 67$ \\
Aortic cross-clamping time, min & $112 \pm 48$ \\
Surgical time, min & $644 \pm 157$ \\
Reconstruction of the ICA & $19(66 \%)$ \\
Number of ICA reconstructions & 35 \\
Average number of ICA reconstructions per case & $1.8 \pm 0.5$ \\
\hline
\end{tabular}

$C P B$ cardiopulmonary bypass, $I C A$ intercostal artery. Data are given as mean \pm standard deviation or frequency (percentage), as appropriate.

patients, significant reductions in amplitude occurred during aortic clamping at T8 to L1. In all patients, the evoked potential amplitude recovered to $>50 \%$ of the baseline value as soon as intercostal and lumbar artery reconstruction was completed and blood pressure was optimized. In eight patients, including two who received open distal anastomosis because aortic clamping was difficult, significant reductions in amplitude occurred at various times. In addition to optimization of blood pressure, initiation of CSFD, and prompt resumption of pelvic or lower limb perfusion, reconstruction of one or two additional intercostal or lumbar arteries was performed in five patients, which was effective in restoring the amplitude of evoked potentials to $>50 \%$ of the baseline value. One patient with a history of open repair for AAA required direct suture repair for a duo- 
denal injury.

\subsection{Hospital Mortality}

No in-hospital deaths occurred among the 29 patients treated during the study period (Table 3).

\subsection{Early Complications}

Table 3 provides a summary of postoperative outcomes. No acute neurological complications such as paraplegia or paraparesis were noted. Delayed paraplegia occurred in one patient (3.4\%). Specifically, a 66-year-old man with a Crawford type II dissecting aneurysm developed delayed paraplegia on postoperative day 2 because of hypotension and CSFD tube occlusion. The patient had undergone reconstruction of two intercostal arteries because of a decrease of $\geq 50 \%$ from the baseline motor-evoked potential during aortic clamping at T8 to L1. However, follow-up contrast-enhanced computed tomography (CT) revealed occlusion of the two reconstructed intercostal arteries. Nevertheless, the patient recovered spontaneously following blood pressure optimization and reinitiation of CSFD, regaining the ability to walk independently.

Infectious complications occurred in four patients (13.8\%). Wound infection was noted in three patients, whereas the fourth patient developed peritonitis with pancreatitis. Chylothorax occurred in three patients (10.3\%). One patient (3.4\%) underwent reoperation because of postoperative bleeding. Other complications such as respiratory failure requiring prolonged mechanical ventilation or tracheotomy, brain infarction, or renal failure requiring new dialysis did not occur.

\subsection{Patency Rate of Reconstructed Intercostal and Lumbar Arteries}

Two of the 19 patients who underwent reconstruction of the intercostal and

Table 3. Postoperative outcomes of open surgery for thoracoabdominal aortic aneurysm.

\begin{tabular}{cc}
\hline Variable & \\
\hline In-hospital death, $\mathrm{n}$ & 0 \\
SCI, n (\%) & $1(3.4 \%)$ \\
Acute & 0 \\
Delayed & $1(3.4 \%)$ \\
Reexploration for bleeding, n (\%) & $1(3.4 \%)$ \\
Chylothorax, n (\%) & $3(10.3 \%)$ \\
Infection, n (\%) & $4(13.8 \%)$ \\
Wound infection & $3(10.3 \%)$ \\
Peritonitis & $1(3.4 \%)$ \\
Respiratory failure requiring new HD, n & 0 \\
Brain infarction, $\mathrm{n}$ & 0 \\
Renal failure, $\mathrm{n}$ & 0 \\
\hline
\end{tabular}

$S C I$ spinal cord injury, $H D$ hemodialysis. 
lumbar arteries could not undergo contrast-enhanced CT because of postoperative renal dysfunction. Therefore, contrast-enhanced CT was used to evaluate the early patency rate in 17 patients (31 reconstructed intercostal and lumbar arteries). The overall patency rate was $35.5 \%$ (11/31).

\subsection{Aorta-Related Events and Late Outcomes}

Table 4 provides a summary of late outcomes. Secondary aortic infection occurred in three patients, each of whom had presented some early postoperative complications due to wound infection, peritonitis with pancreatitis, or duodenum injury. Specifically, a 40-year-old man with Marfan syndrome developed wound infection by Pseudomonas aeruginosa after surgery for a Crawford type II TAAA (Figure 2(A)). Open surgical debridement and lavage using saline were performed, resulting in complete skin closure and discharge on postoperative day 55. However, at 41 months after surgery, the patient presented with prosthetic vascular graft infection by $P$. aeruginosa (Figure $2(B)$ ), which was treated by graft replacement with omental wrapping. A 63-year-old man developed peritonitis with pancreatitis after surgery for a Crawford type II TAAA. Necrosectomy combined with open drainage were performed, and the patient was discharged on postoperative day 249 . However, at 40 months after surgery, the developed a secondary infection at a dilated segment of the abdominal aorta just below the prosthetic vascular graft. The infected dilated abdominal aorta was removed, and the patient received extra-anatomic axillary-bifemoral bypass grafting. Another patient, who had a duodenal injury associated with previous AAA repair, was discharged but developed a graft-duodenal fistula at 6 months

Table 4. Late outcomes of open surgery for thoracoabdominal aortic aneurysm.

\begin{tabular}{cc}
\hline Variable & \\
\hline Secondary aortic infection, $\mathrm{n}(\%)$ & $3(10.3 \%)$ \\
Planned two-stage surgery, n (\%) & $3(10.3 \%)$ \\
Late death, $\mathrm{n}$ & 3 \\
Aorta-related & 1 \\
\hline
\end{tabular}
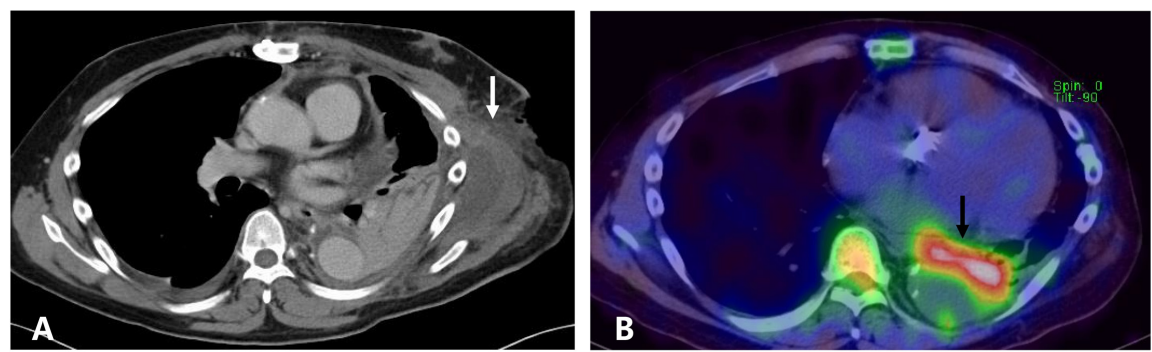

Figure 2. Representative imaging findings in a 40-year-old man who underwent open surgery for a Crawford type II thoracoabdominal aortic aneurysm. (A) Computed tomography scan showing postoperative subcutaneous abscess (white arrow) extending to the chest wall after open drainage. (B) Gallium scintigraphys can showing increased uptake around the graft (arrow). 
after surgery. Due to his poor general medical condition, the patient was treated conservatively but died of sepsis at 21 months after surgery.

Three (10.3\%) patients underwent planned two-stage surgery. Specifically, one patient had a Crawford type II aneurysm and underwent graft replacement in the infra-renal abdominal aorta. Another patient had an infected aneurysm and underwent endovascular repair in the descending thoracic aorta. Finally, a patient with previous history of open repair for AAA underwent endovascular repair for a false aneurysm in the common iliac artery. Other aorta-related events such as rupture of a residual aortic aneurysm, unplanned repeat surgery for residual aortic aneurysm, and infection recurrence after surgery for infected aneurysm did not occur.

Three patients died during the late postoperative period (one aorta-related death, one cardiac death, and one cancer-related death). Figure 3 illustrates the curve describing survival free from aorta-related death. The overall survival rate was $86 \%$, whereas the 3 -year rate of freedom from aorta-related death was $95 \%$.

\section{Discussion}

In TAAA surgery, it is critical to avoid SCI and ischemic damage to the viscera and kidneys, as such complications severely affect prognosis and quality of life. Improved results of open repair for TAAA have been reported for the combination of several important strategies including distal aortic perfusion, moderate hypothermia, sequential aortic clamping, spinal monitoring, CSFD, visceral perfusion, and reconstruction of intercostal and lumbar arteries or AKA [3]-[15]. Despite employing all the above-listed strategies, the outcomes of TAAA operations performed in our hospital before 2011 were quite discouraging, with an $11.1 \%$ rate of in-hospital mortality and an $8.3 \%$ incidence of

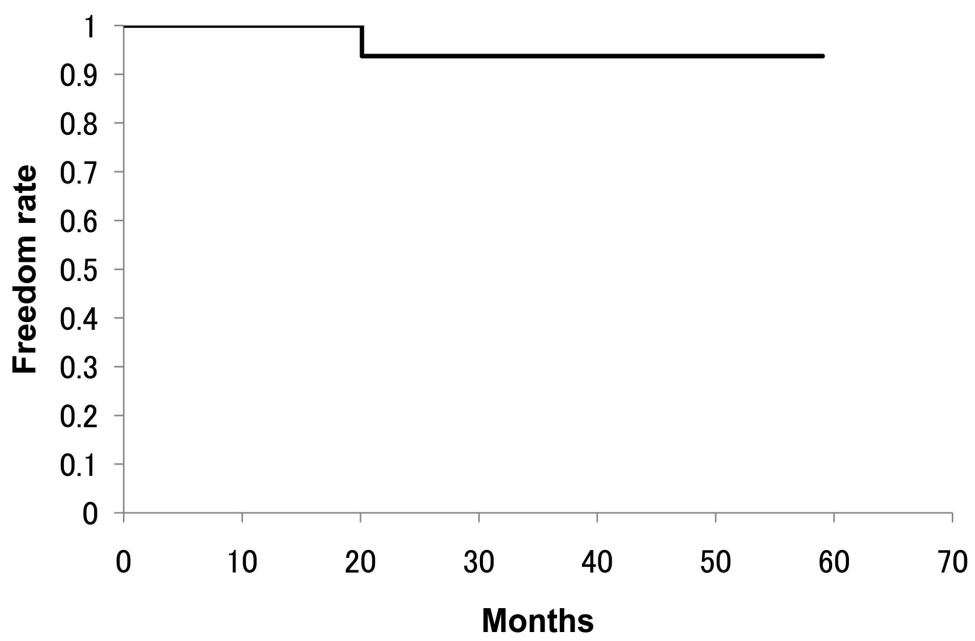

$\begin{array}{llll}\text { Number at risk } & 19 & 12 & 3\end{array}$

Figure 3. Freedom from aorta-related death following open surgery for thoracoabdominal aortic aneurysm. The 3-year rate of freedom from aortic-related death was $95 \%$. 
postoperative paraplegia among the 36 TAAA patients treated during that period. Prior to 2011, we did not attempt to maintain high blood pressure during the operation, did not use motor-evoked potential monitoring of spinal perfusion, and created the last anastomosis in the distal aorta. Since we recognized the importance of maintaining high blood pressure and establishing early pulsatile flow to the spinal cord to prevent spinal cord ischemia [16] [17] [19] [20], we changed our surgical strategy in such a way as to establish pulsatile perfusion to the spinal cord as soon as possible. Specifically, we fabricate a side branch that we anastomose to the main graft at the backtable before intercostal artery reconstruction. Additionally, we create the distal aortic anastomosis before visceral branch reconstruction, in order to perfuse the hypogastric arteries with native pulsatile flow. Finally, we maintain high blood pressure during aortic aneurysm repair and throughout the perioperative period. Since 2011, when we implemented this new surgical strategy, no in-hospital deaths and no acute neurological complications occurred following open surgery for TAAA. Our current study did not collect sufficient data to facilitate statistical analysis of factors influencing the incidence of SCI, and thus we cannot confirm that our new strategy for TAAA treatment is solely responsible for reducing the risk of SCI. Nevertheless, based on our experience, we are convinced that this categorical improvement in the outcomes of TAAA repair was facilitated not only by the gradual increase in the experience of our surgical team, but also by the adoption of this sophisticated surgical strategy that involves maintaining high blood pressure and early establishment of pulsatile flow to the spinal cord.

Despite good overall outcomes, delayed paraplegia occurred in one patient. Currently, most neurologic injuries following TAAA repair have delayed rather than immediate manifestation [20]. Estrera et al. found that the use of combined distal aortic perfusion and CSFD was an independent predictor of delayed neurologic deficit due to SCI in patients who underwent TAAA repair [21]. The authors suggested that such strategies allowed patients to tolerate the initial surgical insult but left them vulnerable to further events such as postoperative CSFD disruption or hypotension, resulting in delayed paraplegia [20]. Our present results support this supposition, as the only patient who developed paraplegia had postoperative hypotension and CSFD tube occlusion.

Furthermore, in the patient with delayed paraplegia, the two reconstructed intercostal arteries were occluded. In this patient series, the overall early patency rate of reconstructed intercostal and lumbar arteries was only $35.5 \%$, which may be at least partly explained by the surgical technique employed for reconstruction of the intercostal arteries. Specifically, we used an $8-\mathrm{mm}$ side graft that had been previously anastomosed to the main graft at the back table. The low patency rate may be related to inadequate sizing of the intercostal artery graft. Shiiya et al. advocated that the patency of reconstructed arteries can be improved by using large-sized $(12-\mathrm{mm})$, separate tube grafts sutured to the aortic wall first and then connected to the main graft in such a way as to minimize graft length 
[15]. Omura et al. reported that the overall patency rate of reconstructed intercostal arteries was significantly lower after graft interposition than after aortic patch anastomosis (65.3\% [147/225] versus 90.8\% [109/120]; p < 0.01) [22]. Other researchers have also confirmed the superior efficacy of techniques involving reconstruction of multiple branches of the intercostal arteries as a single patch over that of creating separate bypass grafts for each vessel [11] [23] [24]. Therefore, a procedure similar to aortic patch anastomosis may be more appropriate than graft interposition in patients without connective tissue disorders, but care should be taken to minimize the patch area for intercostal artery anastomoses in order to prevent late dilatation [22].

There are conflicting views regarding the impact of different strategies for managing intercostal and lumbar arteries or AKA with respect to SCI prevention [11] [12] [13] [15] [20] [25] [26] [27] [28] [29]. While we did not identify the AKA preoperatively, we performed modest reconstruction of the intercostal and lumbar arteries at T8 to L1 according to the reduction in evoked potential amplitude on spinal monitoring [4] [5] [20], a strategy referred to as neuromonitoring-guided repair [20]. However, preoperative identification of the AKA and adequate reconstruction or preservation may help improve spinal cord protection [12]. Afifi et al. also suggested that reconstruction of the T8-T12 intercostal arteries may improve spinal cord perfusion and prevent delayed paraplegia, regardless of intraoperative spinal monitoring data [11].

In this series, no patient required emergent surgery for ruptured aneurysm or acute aortic dissection, and we could perform surgery electively for patients with an infected aneurysm. Moreover, both hypogastric arteries were patent in $97 \%$ of patients even though $24 \%$ of patients had undergone previous AAA repair. These factors likely contributed to the favorable results noted in this series of patients [6] [9] [10] [12].

\section{Limitations}

This was a retrospective study performed over a 6-year period, which enrolled a small number of patients because our hospital is a low-volume center and because the incidence of TAAA is relatively low. Thus, we could not directly compare the results obtained in our small series with those obtained in large series of patients. Further investigations enrolling a larger number of patients are warranted to confirm the current findings.

\section{Conclusion}

In our small-volume hospital, we recently adopted a new surgical strategy for TAAA repair. This novel surgical strategy involves maintaining high blood pressure and early establishment of pulsatile flow to the spinal cord, in combination with other procedures known to improve spinal perfusion, as well as modest reconstruction of the intercostal and lumbar arteries. Although secondary aortic infection occasionally occurred, this novel strategy was safe and effective in pre- 
venting SCI, achieving good early and mid-term results.

\section{Acknowledgements}

We thank Editage (www.editage.jp) for English language editing.

\section{Conflicts of Interest}

The authors declare that they have no conflict of interest.

\section{References}

[1] Shimizu, H., Hirahara, N., Motomura, N., Miyata, H. and Takamoto, S. (2017) Current Status of Cardiovascular Surgery in Japan, 2013 and 2014: A Report Based on the Japan Cardiovascular Surgery Database 5. Thoracic Aortic Surgery. General Thoracic and Cardiovascular Surgery, 65, 671-678.

https://doi.org/10.1007/s11748-017-0822-9

[2] Okita, Y. (2015) Surgery for Thoracicaortic Disease in Japan: Evolving Strategies toward the Growing Enemies. General Thoracic and Cardiovascular Surgery, 63, 185-196. https://doi.org/10.1007/s11748-014-0476-9

[3] Huh, J., Le Mairev, S.A., Weldon, S.A. and Coselli, J.S. (2010) Thoracoabdominal Aortic Aneurysm Repair: Open Technique. Operative Techniques in Thoracic and Cardiovascular Surgery, 15, 70-85. https://doi.org/10.1053/j.optechstcvs.2010.02.002

[4] van Dongen, E.P., Schepens, M.A., Morshuis, W.J., ter Beek, H.T., Aarts, L.P., de Boer, A., et al. (2001) Thoracic and Thoracoabdominal Aortic Aneurysm Repair: Use of Evoked Potential Monitoring in 118 Patients. Journal of Vascular Surgery, 34, 1035-1040. https://doi.org/10.1067/mva.2001.119397

[5] Kawanishi, Y., Munakata, H., Matsumori, M., Tanaka, H., Yamashita, T., Nakagiri, K., et al. (2007) Usefulness of Transcranial Motor Evoked Potentials during Thoracoabdominal Aortic Surgery. The Annals of Thoracic Surgery, 83, 456-461. https://doi.org/10.1016/j.athoracsur.2006.09.056

[6] Le Maire, S.A., Price, M.D., Green, S.Y., Zarda, S. and Coselli, J.S. (2012) Results of Open Thoracoabdominal Aortic Aneurysm Repair. Annals of Cardiothoracic Surgery, 1, 286-292.

[7] Schepens, M.A., Heijmen, R.H., Ranschaert, W., Sonker, U. and Morshuis, W.J. (2009) Thoracoabdominal Aortic Aneurysm Repair: Results of Conventional Open Surgery. European Journal of Vascular and Endovascular Surgery, 37, 640-645. https://doi.org/10.1016/j.ejvs.2009.03.011

[8] Okita, Y., Omura, A., Yamanaka, K., Inoue, T., Kano, H., Tanioka, R., et al. (2012) Open Reconstruction of Thoracoabdominal Aortic Aneurysms. Annals of Cardiothoracic Surgery, 1, 373-380.

[9] Coselli, J.S., Le Maire, S.A., Preventza, O., de la Cruz, K.I., Cooley, D.A., Price, M.D., et al. (2016) Outcomes of 3309 Thoracoabdominal Aortic Aneurysm Repairs. The Journal of Thoracic and Cardiovascular Surgery, 151, 1323-1338. https://doi.org/10.1016/j.jtcvs.2015.12.050

[10] Murana, G., Castrovinci, S., Kloppenburg, G., Yousif, A., Kelder, H., Schepens, M., et al. (2016) Open Thoracoabdominal Aortic Aneurysm Repair in the Modern Era: Results from a 20-Year Single-Centre Experience. European Journal of CardioThoracic Surgery, 49, 1374-1381. https://doi.org/10.1093/ejcts/ezv415

[11] Afifi, R.O., Sandhu, H.K., Zaidi, S.T., Trinh, E., Tanaka, A., Miller 3rd, C.C., et al. 
(2018) Intercostal Artery Management in Thoracoabdominal Aortic Surgery: To Reattach or Not to Reattach? The Journal of Thoracic and Cardiovascular Surgery, 155, 1372-1378. https://doi.org/10.1016/j.jtcvs.2017.11.072

[12] Tanaka, H., Ogino, H., Minatoya, K., Matsui, Y., Higami, T., Okabayashi, H., et al. (2016) The Impact of Preoperative Identification of the Adamkiewiczartery on Descending and Thoracoabdominal Aortic Repair. The Journal of Thoracic and Cardiovascular Surgery, 151, 122-128. https://doi.org/10.1016/j.jtcvs.2015.07.079

[13] Ogino, H., Sasaki, H., Minatoya, K., Matsuda, H., Yamada, N. and Kitamura, S. (2006) Combined Use of Adamkiewicz Artery Demonstration and Motor-Evoked Potentials in Descending and Thoracoabdominal Repair. The Annals of Thoracic Surgery, 82, 592-596. https://doi.org/10.1016/j.athoracsur.2006.03.041

[14] Jacobs, M.J., Mess, W., Mochtar, B., Nijenhuis, R.J., Statius van Eps, R.G. and Schurink, G.W. (2006) The Value of Motor Evoked Potentials in Reducing Paraplegia during Thoracoabdominal Aneurysm Repair. Journal of Vascular Surgery, 43, 239-246. https://doi.org/10.1016/j.jvs.2005.09.042

[15] Shiiya, N., Washiyama, N., Tsuda, K., Yamanaka, K., Takahashi, D., Yamashita, K., et al. (2017) Japanese Perspective in Surgery for Thoracoabdominal Aortic Aneurysms. General Thoracic and Cardiovascular Surgery. https://doi.org/10.1007/s11748-017-0838-1

[16] Kawanishi, Y., Okada, K., Matsumori, M., Tanaka, H., Yamashita, T., Nakagiri, K., et al. (2007) Influence of Perioperative Hemodynamics on Spinal Cord Ischemia in Thoracoabdominal Aortic Repair. The Annals of Thoracic Surgery, 84, 488-492. https://doi.org/10.1016/j.athoracsur.2007.02.089

[17] Izumi, S., Okada, K., Hasegawa, T., Omura, A., Munakata, H., Matsumori, M., et al. (2010) Augmentation of Systemic Blood Pressure during Spinal Cord Ischemia to Prevent Postoperative Paraplegia after Aortic Surgery in a Rabbit Model. The Journal of Thoracic and Cardiovascular Surgery, 139, 1261-1268.

https://doi.org/10.1016/j.jtcvs.2009.08.038

[18] Kise, Y., Kuniyoshi, Y., Inafuku, H., Nagano, T., Hirayasu, T. and Yamashiro, S. (2015) Directly Measuring Spinal Cord Blood Flow and Spinal Cord Perfusion Pressure via the Collateral Network: Correlations with Changes in Systemic Blood Pressure. The Journal of Thoracic and Cardiovascular Surgery, 149, 360-366. https://doi.org/10.1016/j.jtcvs.2014.09.121

[19] Etz, C.D., Di Luozzo, G., Zoli, S., Lazala, R., Plestis, K.A., Bodian, C.A., et al. (2009) Direct Spinal Cord Perfusion Pressure Monitoring in Extensive Distal Aortic Aneurysm Repair. The Annals of Thoracic Surgery, 87, 1764-1774. https://doi.org/10.1016/j.athoracsur.2009.02.101

[20] Estrera, A.L., Sheinbaum, R., Miller, C.C., Harrison, R. and Safi, H.J. (2010) Neuromonitor-Guided Repair of Thoracoabdominal Aortic Aneurysms. The Journal of Thoracic and Cardiovascular Surgery, 140, S131-S135. https://doi.org/10.1016/j.jtcvs.2010.07.058

[21] Estrera, A.L., Miller, C.C., Huynh, T.T., Azizzadeh, A., Porat, E.E., Vinnerkvist, A., et al. (2003) Preoperative and Operative Predictors of Delayed Neurologic Deficit Following Repair of Thoracoabdominal Aortic Aneurysm. The Journal of Thoracic and Cardiovascular Surgery, 126, 1288-1294. https://doi.org/10.1016/S0022-5223(03)00962-0

[22] Omura, A., Yamanaka, K., Miyahara, S., Sakamoto, T., Inoue, T., Okada, K., et al. (2014) Early Patency Rate and Fate of Reattached Intercostal Arteries after Repair of Thoracoabdominal Aortic Aneurysms. The Journal of Thoracic and Cardiovascular Surgery, 147, 1861-1867. https://doi.org/10.1016/j.jtcvs.2013.06.035 
[23] Zhang, L., Sun, X.G., Yu, C.T., Chang, Q. and Qian, X.Y. (2016) Intercostal Artery Reconstruction: The Simple and Effective Technique on Spinal Cord Protection during Thoracoabdominal Aortic Replacement. Annals of Vascular Surgery, 34, 62-67. https://doi.org/10.1016/j.avsg.2015.12.030

[24] Woo, E.Y., Mcgarvey, M., Jackson, B.M., Bavaria, J.E., Fairman, R.M. and Pochettino, A. (2007) Spinal Cord Ischemia May Be Reduced via a Novel Technique of Intercostal Artery Revascularization during Open Thoracoabdominal Aneurysm Repair. Journal of Vascular Surgery, 46, 421-426.

https://doi.org/10.1016/j.jvs.2007.04.048

[25] Griepp, R.B. and Griepp, E.B. (2007) Spinal Cord Perfusion and Protection during Descending Thoracic and Thoracoabdominal Aortic Surgery: The Collateral Network Concept. The Annals of Thoracic Surgery, 83, S865-S869.

https://doi.org/10.1016/j.athoracsur.2006.10.092

[26] Minatoya, K., Karck, M., Hagl, C., Meyer, A., Brassel, F., Harringer, W., et al. (2002) The Impact of Spinal Angiography on the Neurological Outcome after Surgery on the Descending Thoracic and Thoracoabdominal Aorta. The Annals of Thoracic Surgery, 74, S1870-S1878. https://doi.org/10.1016/S0003-4975(02)04149-8

[27] Estrera, A.L. (2016) The Artery of Adamkiewicz: More Interesting than Practical? The Journal of Thoracic and Cardiovascular Surgery, 151, 129-130. https://doi.org/10.1016/j.jtcvs.2015.08.028

[28] Etz, C.D., Kari, F.A., Mueller, C.S., Silovitz, D., Brenner, R.M., Lin, H.M., et al. (2011) The Collateral Network Concept: A Reassessment of the Anatomy of Spinal Cord Perfusion. The Journal of Thoracic and Cardiovascular Surgery, 141, 1020-1028. https://doi.org/10.1016/j.jtcvs.2010.06.023

[29] Etz, C.D., Halstead, J.C., Spielvogel, D., Shahani, R., Lazala, R., Homann, T.M., et al. (2006) Thoracic and Thoracoabdominal Aneurysm Repair: Is Reimplantation of Spinal Cord Arteries a Waste of Time? The Annals of Thoracic Surgery, 82, 1670 1677. https://doi.org/10.1016/j.athoracsur.2006.05.029

\section{Abbreviations}

TAAA: Abdominal Aortic Aneurysm;

SCI: Spinal Cord Injury;

CPB: Cardiopulmonary Bypass;

CSFD: Cerebrospinal Fluid Drainage;

AKA: Adamkiewicz Artery;

AAA: Abdominal Aortic Aneurysm;

CT: Computed Tomography. 